\title{
Shock Temperature Measurements in Metals: New Results for an Fe Alloy
}

\author{
Jay D. Bass ${ }^{1}$, Thomas J. Ahrens ${ }^{2}$, John R. Abelson ${ }^{3}$ and Tan Hua ${ }^{2,4}$
}

\begin{abstract}
The temperature of a $\mathrm{Fe}-\mathrm{Cr}-\mathrm{Ni}$ alloy (304 stainless steel) has been measured during shock compression using a high-speed radiometric technique. Experiments were performed on highquality thick films deposited on sapphire and LiF windows. The samples had no observable porosity or defects and closely meet the ideal criteria for shock temperature measurements. Data obtained with both $\mathrm{Al}_{2} \mathrm{O}_{3}$ and $\mathrm{LiF}$ windows are internally consistent, indicating that they remain transparent to high pressures and are thus suitable windows for shock temperature measurements. Our data yield stainless steel melting temperatures ranging from $4570 \pm 310 \mathrm{~K}$ at $138 \mathrm{GPa}$ to $5710 \pm 340 \mathrm{~K}$ at $215 \mathrm{GPa}$, and additionally provide bounds on the initial Hugoniot temperatures of the sample between $5600 \pm 340 \mathrm{~K}$ at $234 \mathrm{GPa}$ (near the solidus) and $6580 \pm 440 \mathrm{~K}$ at 283 (in the liquid field). Taken together, these data define a smooth curve for melting of the alloy up to 271 $\mathrm{GPa}$ and $5860 \mathrm{~K}$, which should represent a point on the liquidus. Melting along the Hugoniot begins at approximately $234 \mathrm{GPa}$ and $5600 \mathrm{~K}$, as compared with $242 \mathrm{GPa}$ and $6400 \mathrm{~K}$ for pure $\mathrm{Fe}$. At the pressure of the inner core-outer core boundary, the melting point of 304 stainless steel is lower than that of pure Fe by $\approx 1450 \mathrm{~K}$, as compared with only $110 \mathrm{~K}$ at 1 atm. These results demonstrate that upon alloying with $\mathrm{Ni}$ and $\mathrm{Cr}$ the melting point depression of $\mathrm{Fe}$ and thus material likely to comprise the inner core increases with increasing pressure.
\end{abstract}

\section{INTRODUCTION}

Studies of the melting relations in metallic systems at high pressures have direct implications for the thermal structure and composition of the Earth's core and lower mantle. Because the primary constituent of the liquid outer core is believed to be an iron alloy, the high-pressure melting behavior of Fe-bearing compounds are of singular importance for understanding the nature of the innermost portions of the Earth. In particular, the temperature of the inner coreouter core boundary, at a pressure of $329 \mathrm{GPa}$ [Dziewonski and Anderson, 1981], is controlled by the melting point of the iron alloy at this pressure. With the development of new techniques in the areas of shock wave research, the pressure range over which melting phenomena have been studied has greatly expanded, thus offering the potential for constructing improved models of the Earth's interior.

Shock wave methods have long played a particularly important role in studies of core materials due to the extreme temperatures and pressures that are accessible, thereby allowing properties to be measured under conditions similar to those in the core itself. The temperature achieved under shock conditions is not, however, a readily measured variable, and this has been a serious limitation to the application of shock wave results. Although the possibility of measuring shock temperatures in opaque materials such as metals has been recognized for some time [Urtiew, 1974; Urtiew and

\footnotetext{
1 Department of Geology, University of Dlinois, Urbana.

2 Seismological Laboratory, California Institute of Technology, Pasadena.

3 Coordinated Sciences Laboratory, University of Illinois, Urbana.

${ }^{4}$ Permanently at Beijing Institute of Technology, Beijing, Peoples Republic of China.
}

Copyright 1990 by the American Geophysical Union.

Paper number 90JB02163.

0148-0227/90/90JB-02163\$05.00
Grover, 1977; Lyzenga and Ahrens, 1979], the experiment is difficult in both execution and interpretation. Specifically, the ideal shock temperature experiment on metals places stringent requirements on the sample assembly used. Only recently has there been a sustained effort to measure the temperature of shocked opaque solids. Bass et al. [1987] carried out a series of temperature measurements on iron at Hugoniot pressures up to $300 \mathrm{GPa}$, close to the pressure at the inner core-outer core boundary. These results were found to be in close agreement with static compression measurements of melting temperatures in $\mathrm{Fe}$ at lower pressures by Williams et al. [1987] and with theoretical Hugoniot temperatures calculated by McQueen et al. [1970]. However, these results yielded higher melting temperatures than those obtained by the theoretical calculations of Brown and McQueen [1986], and the diamond anvil experiments of Boehler [1986] and Boehler et al. [this issue]. In this report we review the basis of shock temperature measurements on metals and recent developments in the analysis of such data, and we present new data on shock temperatures measured on an $\mathrm{Fe}-\mathrm{Cr}-\mathrm{Ni}$ alloy. The experiments were performed on samples of extremely high quality, thereby eliminating some of the uncertainties that were present in the previous data on Fe. Our results constrain the melting curve of the alloy over a pressure range of about 138-271 GPa and are consistent with a melting point depression of $\mathrm{Fe}$ due to alloying with $\mathrm{Ni}$ and $\mathrm{Cr}$. These new results support our previous conclusions on the melting curve of $\mathrm{Fe}$ at core pressures.

\section{EXPERIMENTAL MethodS}

\section{Shock Temperature Measurements}

Although the optical pyrometric technique used in our shock temperature measurements has been presented previously [Lyzenga and Ahrens, 1979; Bass et al., 1987; Boslough and Ahrens, 1989], a brief description of the experiment is given as background for the following discussion. The temperature $T$ of a sample shocked to high pressure $P$ is obtained by measuring the intensity of thermal radiation emit- 
ted by the sample at several discrete wavelengths $\lambda$. The temperature and emissivity $\epsilon$ of the sample are obtained by fitting the observed spectral data to a Planck greybody radiation function

$$
L(\lambda)=\epsilon C_{1} \lambda^{-5}\left[\exp \left(C_{2} / \lambda T\right)-1\right]^{-1}
$$

where $L(\lambda)$ are the spectral radiances and $C_{1}$ and $C_{2}$ are constants with values of $1.191 \times 10^{-16}$ and $1.439 \times 10^{-2}$, respectively. The challange and complications of performing such an experiment on a metal arise from the simple fact that metals are opaque, and it is possible to collect radiation emitted only from the surface of a sample and not from the interior. Because the shock pressure releases to $1 \mathrm{~atm}$ at a free surface and is accompanied by an adiabatic decrease in $T$, it is necessary to maintain the sample at high $P$ and $T$ by means of a transparent anvil in contact with the sample surface. The anvil material also serves as a window through which the thermal radiation must be transmitted (Figure 1). In contrast, a transparent sample serves as its own anvil and window. An overriding consideration in the choice of anvil/window material is, therefore, that it remain transparent, or nearly so, up to the pressures of interest. For work in the megabar pressure range and above, sapphire (single-crystal $\mathrm{Al}_{2} \mathrm{O}_{3}$ ) and $\mathrm{LiF}$ are the only known suitable window materials.

It is generally not possible to find an anvil with properties that match the shock impedence and thermal characteristics of the sample. Therefore, upon arrival of a shock wave at the sample-anvil interface the pressure will either partially release to a lower value or be reshocked up to higher $P$. In

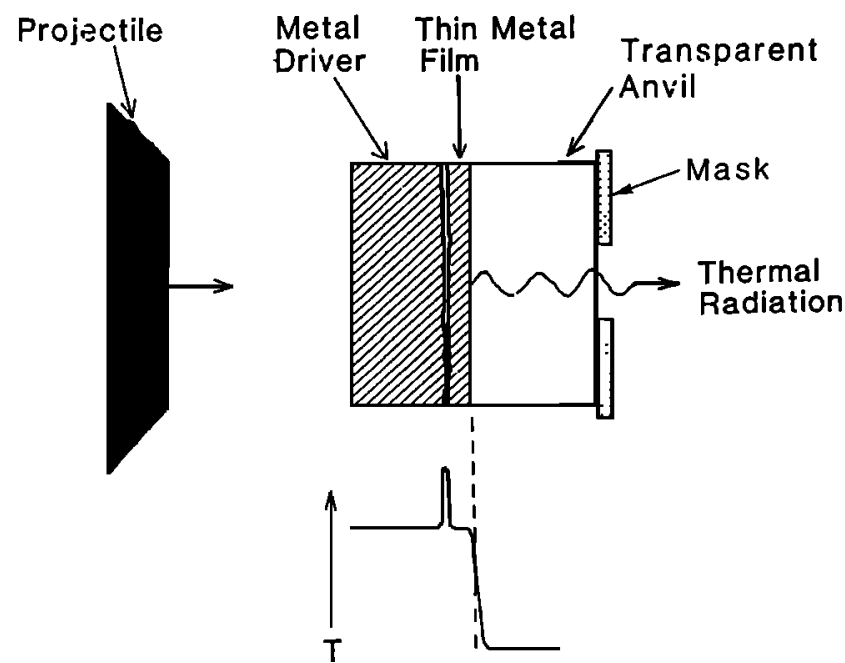

Fig. 1. Sample assembly for optical shock temperature measurements. The sample consists of a metal film deposited on a transparent substrate which serves as both an anvil and a transparent window through which thermal radiation is emitted. Rapid compression of gasses and surface irregularities at the interface between the sample film and the driver produce very high temperatures in this region. The bottom portion of the figure illustrates the thermal distribution across through the assembly [after Bess et al., 1987].

addition, there is an exchange of heat between the sample and anvil due to the different Hugoniot temperatures attained in each of these materials. The magnitudes of these effects are in fact substantial and must be accounted for in the data reduction. We discuss these effects in more detail below.

In the design and preparation of sample assemblies, the objective is to avoid any sources of spurious thermal radiation which would contaminate the observed signal. The most obvious imperfections are sample porosity and spaces, or gaps, between the sample and anvil. At a given Hugoniot pressure an initially porous sample attains higher temperatures than a sample of ideal crystal density. Sample material adjacent to a gap is multiply shocked and also reaches anomalously high temperatures. In addition, it is desirable for the samples to be as thick as possible. Irregularities at the driver-sample interface can lead to a high temperature in this region (shown schematically at the bottom of Figure 1), and this thermal "spike" can possibly conduct to the sampleanvil interface during a measurement. Interactions at this interface have been analyzed by approximating irregularities in the surfaces as thin porous layers which reach very high shock temperatures [Urtiew and Grover, 1974]. Reasonable as this approximation may appear, it should be noted that the validity of this model has not been demonstrated, and it is unclear (as noted by Urtiew and Grover [1974]) how a given state of surface roughness might be quantitatively equated with a specific degree of porosity. In any event, the problem of surface roughness is minimized, if not eliminated, by using vapor deposited films, as opposed to mechanically polished samples placed in contact with the anvil. Electron micrographs of vapor-deposited Fe films showed the roughness to be of the order of the microscope resolution $(\approx 0.02 \mu \mathrm{m}$ ) [Ahrens et al., 1990a], approximately 2 orders of magnitude smaller than the cases examined by Urtiew and Grover [1974]. Although roughness of the film surface should therefore have a minimal effect on the observed temperatures, we have made a considerable effort to obtain thick films in order to thermally isolate the sample-anvil interface from any driver-sample effects.

\section{Samples}

Stainless steel films were deposited on sapphire and LiF substrates using planar magnetron sputtering in an argon atmosphere. The composition of the sputtered samples was determined by electron probe microanalysis to be nearly identical to the 304 stainless steel used in the equation of state measurements of $M c Q u e e n$ et al. [1970], with $C_{r}$ and $\mathrm{Ni}$ as the primary elements alloying with $\mathrm{Fe}$ (Table 1). Using a standard micrometer, the thicknesses of several films were measured and found to be in the range 12-14 $\pm 2 \mu \mathrm{m}$. It is noteworthy that these films were approximately an order of magnitude thicker than some of the Fe films used by Bass et al. [1987], thereby reducing possible contamination of the thermal signal by heat from the driver-anvil interface. Adhesion of the films to the anvil substrates was excellent; no gaps could be seen at the anvil-sample interface by visual observation with and without an optical microscope, and there were no interference fringes upon illumination of the interface with monochromatic radiation. Moreover, the surface of the films had a mirror-like appearance and no observable roughness. The state of the film material was that of a fine polycrystalline aggregate with no obvious intergranular spaces.

Because porosity can greatly affect the Hugoniot temperatures, it is important to accurately characterize the density of films used in shock temperature experiments. The 
TABLE 1. Chemical Composition of Samples

\begin{tabular}{lcc}
\hline & $\begin{array}{c}\text { Stainless Steel } \\
\text { Film }\end{array}$ & $\begin{array}{c}\text { Type 304 Stainless } \\
\text { [McQueen et al., 1970] }\end{array}$ \\
\hline $\mathrm{Fe}$, wt \% & 69.3 & 68.0 \\
$\mathrm{Cr}$ & 19.4 & 19.0 \\
$\mathrm{Ni}$ & 9.1 & 10.0 \\
$\mathrm{Mn}$ & - & 2.0 \\
$\mathrm{Si}$ & 0.7 & 1.0 \\
$\mathrm{C}$ & - & 0.08 \\
Total & 98.5 & 100.08 \\
& & 7.896 \\
\hline
\end{tabular}

Archemedian density of our films was measured using a temperature-monitored toluene bath. The room temperature initial density obtained was $7.82 \pm 0.08 \mathrm{Mg} / \mathrm{m}^{3}$ for the stainless steel films. This value is in agreement with the value of $7.896 \mathrm{Mg} / \mathrm{m}^{3}$ reported by $M c Q$ ueen et al. [1970]. It should be noted that a primary source of error in the density measurements is surface tension of the hanger used to support the sample, an effect which tends to systematically underestimate the sample density. Calibration runs using sapphire and quartz chips of approximately the same mass as the foils yielded results which were on average about $0.5 \%$ lower than the true densities. The results of our measurements indicate that the film samples are essentially of ideal bulk crystal density.

\section{Shock Wave Measurements}

All of the shock temperature measurements were performed using a two-stage light-gas gun [Jeanloz and Ahrens, 1977]. Tantalum flyer plates mounted in Lexan projectiles were accelerated to velocities of between 5.884 and 6.048 $( \pm 0.002) \mathrm{km} / \mathrm{s}$ and impacted Fe driver plates on the target assemblies. The pressure in the sample was calculated by the impedence-matching method [Rice et al., 1958], using the equation of state parameters given by Mitchell and Nellis [1981], Brown and McQueen [1986], Carter [1973], Marsh [1980], and McQueen et al. [1970] for Ta, Fe, LiF, $\mathrm{Al}_{2} \mathrm{O}_{3}$, and 304 stainless steel, respectively. Thermal radiation emitted by the sample was focused onto four photodiodes in front of which were interference filters centerd at wavelengths of $450,600,750$, and $900 \mathrm{~nm}$. A mask was used so that only light from the central area of the sample reached the pyrometer, and light from the edges of the sample was blocked. Prior to each experiment the pyrometer was calibrated using a tungsten filament quartz halogen lamp which serves as a spectral irradiance standard and which is itself calibrated relative to a National Bureau of Standards source. The spectral data were recorded by either high-speed Textronix oscilloscopes (shots 214 and 215) or Hewlett-Packard Model 54111D digital oscilloscopes. In addition, a LeCroy Model $7600 \mathrm{~A}$ digital recording system was used as a backup system. Further details of the temperature measuring system are given by Boslough and Ahrens [1989].

\section{Results}

Three experiments were conducted using sample assemblies of steel deposited on sapphire, and one was performed using LiF as the window/anvil material. Because the shock impedences of $\mathrm{Al}_{2} \mathrm{O}_{3}$ and $\mathrm{LiF}$ are lower than that of stainless steel, a rarefaction, or release wave was propagated back into the sample upon arrival of the initial shock wave at the sample-anvil interface. Pressures corresponding to both the initial Hugoniot state and partially released state of the sample are listed in Table 2.

The raw data from our experiments consist of voltage as a function of time, with the voltage proportional to the intensity of thermal radiation. The source of the radiation is the surface of the partially released sample which is in thermal contact with a relatively cool anvil. As discussed below, models of Fourier heat transport suggest a temperature for this interface which is time independent [Grover and Urtiew, 1974; Tan and Ahrens, 1990]. However, we observed an increase in voltage at later times of all shot records, even if the initial part of the shot record appeared flat (Figure 2). A time dependence of the thermal signal in sapphire windows was similarly observed by Urtiew [1974], and $M c$ Queen and Issak [this issue]. This could indicate that the actual thermal interactions near the sample-window interface are more complex than indicated by theoretical models thus far proposed, and that the temperature at this interface will change with time even for perfect sample assemblies. Alternatively, the rise in voltage could conceivably be due to radiation from the layer of shocked window material which thickens with time [Svendsen et al., 1989]. Yet a third possibility is that heat from the driver-sample interface is diffusing to the sample-window interface on the time scale of the experiment, despite the thickness of the films. Due to the difficulties of interpreting later portions of the shot records, we use the early part of the record, immediatly after the initial rise time, for the purpose of obtaining shock temperatures. These data should represent an interface temperature viewed through unshocked window material, before heat has diffused far from the interfaces. Although complete analyses of the time evolution of the thermal signal will be an important area of investigation in future shock temperature studies, we limit ourselves for the present to a more straightforward interpretation of the initial observable interface radiation.

It should be noted that none of the stainless steel records showed an initial spike or sharp peak in radiant intensity. Such a feature would indicate a high transient temperature from either a poor interface with gaps between the sample and window or a "flash" from compression of gasses and surface irregularities at the driver-sample interface. It is possible, however, that evidence of a thermal transient could have been obscured in some of our shots due to relatively long rise times $(\approx 100 \mathrm{~ns})$. The rise time was sometimes lengthened (as for shot 218 shown in Figure 2), by tilt of the projectile which resulted in non-planar impact onto the target. This tilt was clearly evident on $X$ radiographs of the projectile in flight and was correlated with the observed rise time from oscillograph traces of the light intensity. Nontheless, even for shots with much shorter rise times of $\sim 20$ ns or less (approximately the response time of the detection system), there was no indication of a thermal spike. We thus conclude that our samples were sufficiently thick so that the initial observed radiation was uncontaminated by processes at the driver-sample interface.

It has sometimes been suggested that any time dependence of the voltage is a sign of the window materials becoming opaque. In this event, however, the temperature should approach that of the window material at the release 
TABLE 2. Shot Summary: 304 Stainless Steel

\begin{tabular}{|c|c|c|c|c|c|c|c|c|}
\hline Shot & Anvil & $\begin{array}{l}\text { Velocity } \\
\mathbf{k m} / \mathbf{s}\end{array}$ & $\begin{array}{c}P_{H} \\
\text { GPa }\end{array}$ & $\begin{array}{c}P_{R} \\
\mathbf{G P a}\end{array}$ & $\begin{array}{c}T_{I}, \mathrm{~K} \\
\text { observed }\end{array}$ & $T_{M}, \mathbf{K}$ & $T_{H}, \mathbf{K}$ & $\begin{array}{c}\epsilon \\
\text { observed }\end{array}$ \\
\hline $\begin{array}{l}218 \\
219 \\
214 \\
215\end{array}$ & $\begin{array}{l}\mathrm{Al}_{2} \mathrm{O}_{3} \\
\mathrm{LiF} \\
\mathrm{Al}_{2} \mathrm{O}_{3} \\
\mathrm{Al}_{2} \mathrm{O}_{3}\end{array}$ & $\begin{array}{l}5.480 \\
5.884 \\
6.048 \\
6.218\end{array}$ & $\begin{array}{l}234 \\
260 \\
271 \\
283\end{array}$ & $\begin{array}{l}181 \\
138 \\
204 \\
215\end{array}$ & $\begin{array}{l}4538 \pm 40 \\
4243 \pm 110 \\
4739 \pm 130 \\
5342 \pm 140\end{array}$ & $\begin{array}{l}5130 \pm 240 \\
4570 \pm 310 \\
5160 \pm 330 \\
5710 \pm 340\end{array}$ & $\begin{array}{l}5600 \pm 340 \\
5630 \pm 410 \\
5860 \pm 430 \\
6580 \pm 440\end{array}$ & $\begin{array}{l}0.39 \pm .02 \\
0.17 \pm .02 \\
0.48 \pm .06 \\
0.38 \pm .04\end{array}$ \\
\hline
\end{tabular}

$T_{I}$ is the observed temperature of the interface between the stainless steel foil and the anvilwindow substrate; $T_{M}$ is the melting temperature of stainless steel at pressure $P_{R}$ inferred from the analysis of $T a n$ and Ahrens, [1990]; $T_{H}$ is the inferred Hugoniot temperature of stainless steel using the analysis of Grover and Urtiew [1974], which ignores melting in the sample and/or anvil.

pressure. Theoretical Hugoniot temperatures, calculated using the procedure of Ahrens [1987], indicate that the window is at a much lower temperature than the metal sample and, therefore, that the voltage should decrease with time, not increase as is observed. In several other investigations of the optical properties of potential window materials, it has similarly been concluded that single-crystal $\mathrm{Al}_{2} \mathrm{O}_{3}$ and $\mathrm{LiF}$ remain transparent to high Hugoniot pressures; these include a reanalysis of Urtiew's [1974] temperature measurements on $\mathrm{Mg}$ [Ahrens et al., 1990a; McQueen and Issak, this issue], experiments on Fe [Bass et al., 1987; Svendsen et al., 1989], and measurements by McQueen and Isaak [this issue] using a variety of sample materials.

Spectral radiances at four wavelengths were fit by a weighted least-squares method to equation (1) in order to obtain the effective emissivity and temperature of the interface (Table 2). The quality of the fits was generally excellent (Figure 3), with uncertainties in temperature being $140 \mathrm{~K}$ or less (one standard deviation), for all shots. In the data reduction, the weighting factor for each pyrometer channel included uncertainties in calibration as well as those related to the reading of shot records. Little difference in the results was obtained by using an unweighted fitting procedure.

Our interpretation of the radiance data necessarily assumes that there is no wavelength dependence to the emissivity of the sample. Although this assumption could have a significant effect on the resultant temperatures, it is common to all measurements of temperatures by optical pyrometry, regardless of whether the measurements are made under dynamic or static conditions in a diamond cell. The only alternative at present is to assume a wavelength dependent emissivity identical to, for example, that of tungsten (one of the few materials for which $\epsilon(T)$ has been measured). There is no clear basis for preferring one of these assumptions over the other. Furthermore, we assume that the metallic sample is radiating homogeneously and that no "hot spots" or

Shot \#218: 304 Stainless Steel on $\mathrm{Al}_{2} \mathrm{O}_{3}$

$$
\begin{aligned}
& \lambda=900 \mathrm{~nm} \\
& P_{H}=234 \mathrm{GPa}, T_{I}=4538 \mathrm{~K}
\end{aligned}
$$

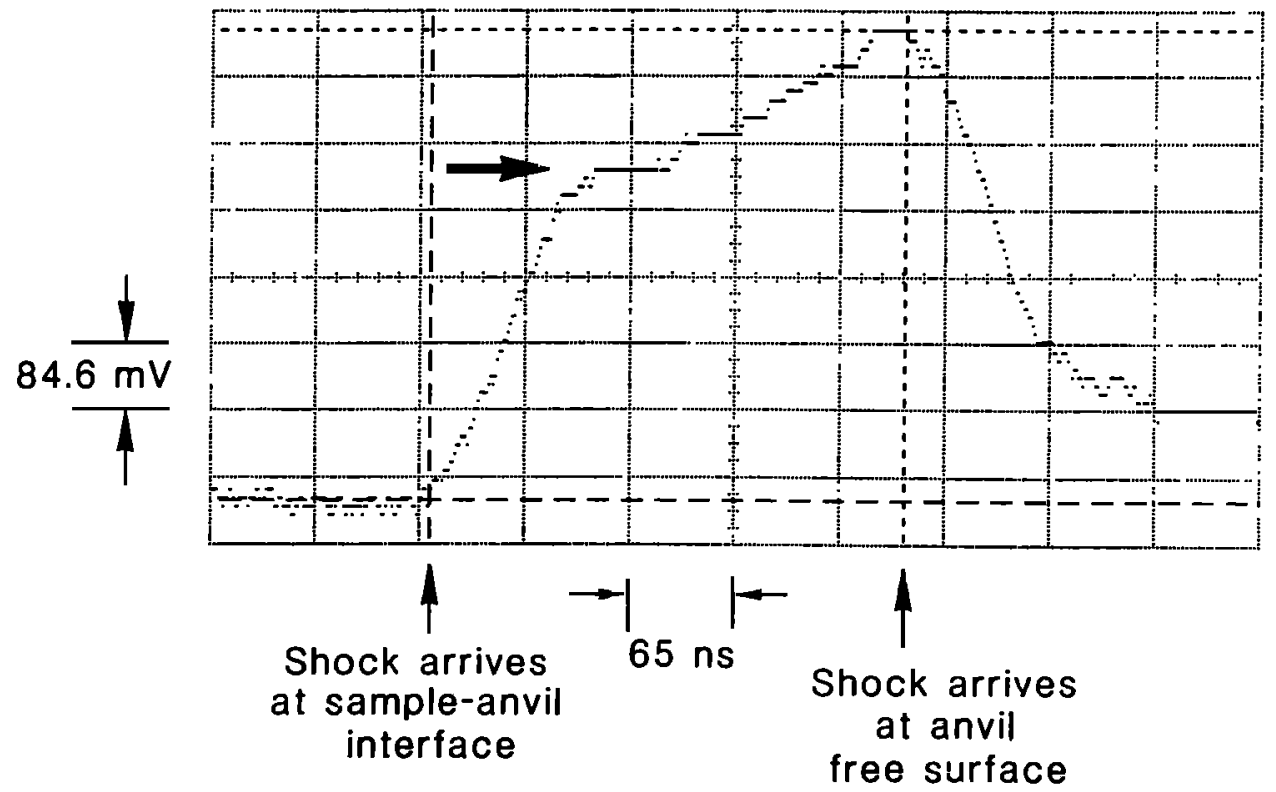

Fig. 2. Digital oscilloscope record of a shock temperature experiment (shot 218). The sample is a 304 stainless steel film on a window/anvil material of $\mathrm{Al}_{2} \mathrm{O}_{3}$. The horizontal arrow on the record indicates the voltage which was used in the data reduction to obtain shock temperatures. 


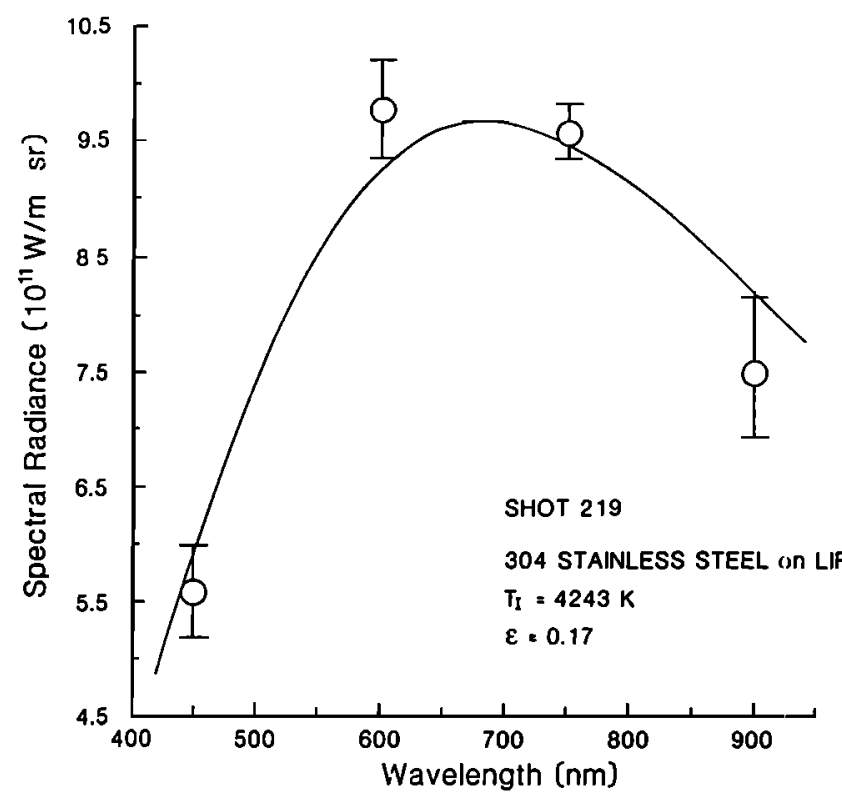

Fig. 3. The optical data measured for a shock temperature experiment using a stainless steel film on a LiF window. The solid curve is a weighted least squares fit to the data using a Planck greybody function.

"shear bands" (localized regions of high temperatures) exist in the sample. This assumption is justified on the basis of lower-pressure shock wave studies which show that shear band deformation characteristically yields high temperatures and low emissivities that are at least one order of magnitude lower than those obtained so far in shock temperature experiments on metals [Schmitt and Ahrens, 1984]. It will be important to test this assumption in the future by high-speed framing photography of the radiating sample.

\section{Discussion}

As originally conceived, optical radiometric measurements on shocked opaque materials could be used as a means of obtaining Hugoniot temperatures $T_{H}$. Anticipating the results of the following discussion, it is also possible under certain conditions to infer the melting temperatures $T_{M}$ of a metal over a range of release pressures $P_{R}$ [Tan and Ahrens, 1990]. We show below that values of $T_{M}$ and $T_{H}$ from our stainless steel data are internally consistent and are inferred to constrain the melting temperatures of 304 stainless steel at pressures between approximately 138 and $271 \mathrm{GPa}$.

Although the metallic sample in a shock temperature experiment is, by virtue of its contact with an anvil, in a partially released and cooled state during a measurement, the effects of pressure release and thermal conduction near the interface can be accounted for. The isentropic release temperatures, $T_{R}$, are related to Hugoniot temperatures by

$$
T_{R}=T_{H} \exp -\int_{V_{H}}^{V_{R}} \frac{\gamma}{V} d V
$$

where $V_{H}$ and $V_{R}$ are the Hugoniot and released volumes, respectively, and $\gamma$ is the Grüneisen parameter. $V_{R}$ is obtained via an approximation to the Riemann integral formula [Lyzenga and Ahrens, 1978]. This approximation yie-
Ids a lower bound on the release volume and release temperature.

The question of heat exchange by the window and sample was first investigated by Grover and Urtiew [1974]. These authors concluded that the interface temperature $T_{I}$ should be time independent and is given by

$$
T_{I}=T_{R}+\frac{\left(T_{a}-T_{R}\right)}{(1+\alpha)}
$$

where subscript $a$ refers to the transparent anvil material. $\alpha$ in equation ( 3 ) is defined as

$$
\alpha=\left(\frac{\kappa_{R} \rho_{R} C_{R}}{\kappa_{a} \rho_{a} C_{a}}\right)^{1 / 2}
$$

where $\kappa$ is the thermal conductivity, $\rho$ is the density, and $C$ is the specific heat.

A point of concern in the evaluation of shock temperature results is the error in $T_{H}$ introduced by uncertainties in thermal properties, particularly $\kappa$. However, it is important to bear in mind that these thermal properties are used to obtain a temperature correction, which is much smaller than the observed temperatures. As an example, consider the effect of errors in $\kappa$ on the inferred $T_{H}$ of Fe, using the data for shot 189 of Bass et al. [1987] at $P_{H}=202 \mathrm{GPa}$ and $T_{I}=4010 \mathrm{~K}$. Using the data and method given by $T a n$ and Ahrens [1990] to calculate the necessary thermal properties, we obtain a value of $\alpha=4.4$. Allowing $\alpha$ to vary by a factor of 2 changes the calculated $T_{H}$ by $\pm 250 \mathrm{~K}$. This is a rather extreme variation in $\alpha$ which is chosen to overestimate likely errors in this parameter. Moreover, related data are available to constrain the thermal properties. The electrical conductivity $\sigma$ of Fe under shock compression has been measured [Keeler, 1971; Matassov, 1977], and this is related to $\kappa$ by the Wiedemann-Franz relation $\kappa=L \sigma T$, where $L$ is the Lorenz number [Ashcroft and Mermin, 1976]. A Debye-Grüneisen model, which is in good agreement with available data on alkalai halides and minerals [Roufosse and Jeanloz, 1983], can be used to estimate the pressure dependence of $\kappa$ for window materials [Bass et al., 1987]. The heat capacity of anvil materials can be approximated by the high temperature DuLong-Petit value, whereas possible electronic contributions to $C$ for Fe and its simple alloys has been investigated theoretically by Boness et al. [1986]. It is unlikely that our calculations of thermal properties would be cooperatively biased in such a way as to yield an error in $T_{H}$ that is decidedly nonrandom.

The use of equations (2)-(4) has been applied to a majority of shock temperature measurements made by optical radiometry [Urtiew and Grover, 1977; Lyzenga and Ahrens, 1979; Bass et al., 1987; McQueen and Issak, this issue]. However, it has usually been assumed that the sample does not undergo any phase transitions, in particular melting, upon partial release of pressure. When the initial Hugoniot state is in the vicinity of the melting curve, release melting can have a significant effect on the interpretation of observed interface temperatures [Tan and Ahrens, 1990]. A schematic illustration of the release melting process is shown in Figure 4, where a material releases sufficiently far from an initial Hugoniot state that the release isentrope intersects the melting curve (point 2). Thereafter, the release path follows the melting curve with the production of an increasing amount 


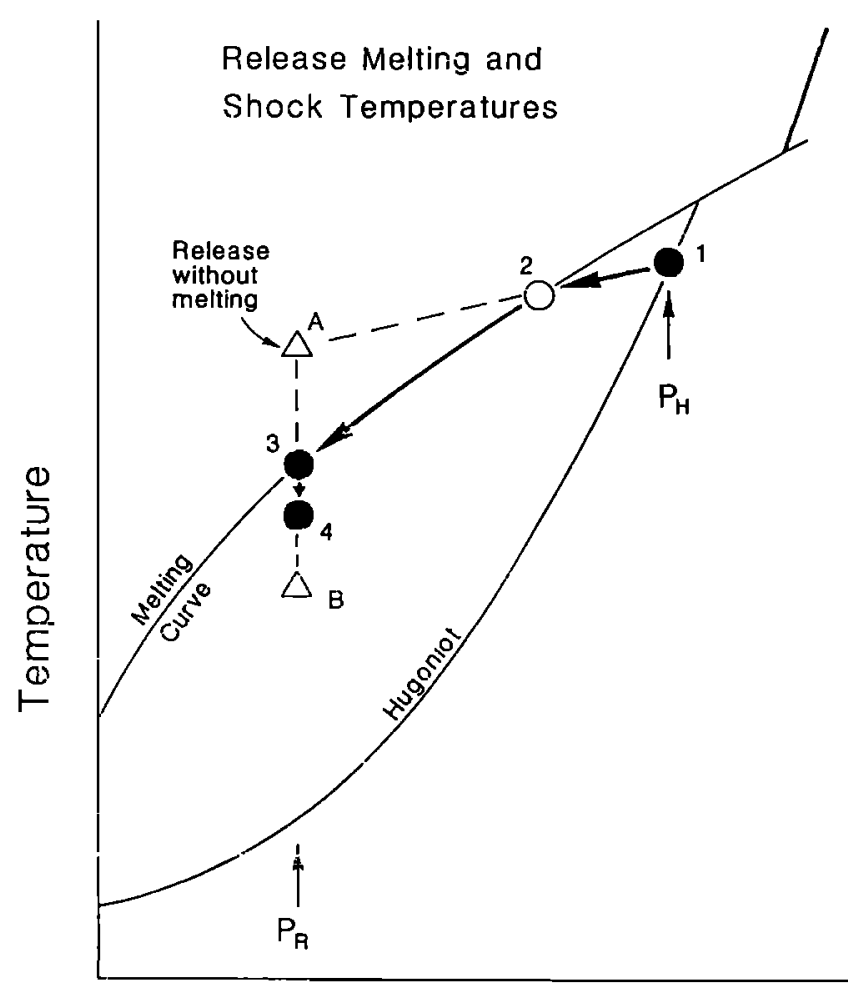

Pressure

Fig. 4. A schematic diagram illustrating the T-P path followed by a sample that melts upon partial release of pressure. The sample is initially shocked to state 1 at pressure $P_{H}$ on the Hugoniot and releases to pressure $P_{R}$ when the shock arrives at the sampleanvil interface. The release isentrope intersects the melting curve at point 2 and follows it to $P_{R}$ (point 3 ). The ratio of melt to solid increases between points 2 and 3 . Without partial melting the final release state would be point $A$. Contact with a cold anvil causes the temperature to drop from point 3 to 4 and is accompanied by freezing of melted sample. The temperature decrease without freezing of melt is much greater (points A to B) due to the latent heat of fusion. The final state of the sample is thus buffered by the melting curve.

of melt and a decrease in temperature which is determined by the thermodynamic properties of the phases. In Figure 4 the final release state is at point 3 on the melting curve, indicating that the sample did not completely melt upon release. For comparison, without any melting the sample would have released to point $A$. Consider further that if the partially released sample is then placed in contact with a relatively cold anvil, the temperature does not decrease until freezing of the melt is complete, due to the latent heat of fusion. The temperature decrease due to heat conduction (points 3 and 4, Figure 4) is therefore much smaller than it would be in the absence of a melting transition (points $A$ and $B$, Figure 4). The central point to be made is that the final state of the sample is either on the melting curve or very close to it. That is, the melting curve essentially buffers the state of the sample, and the interface temperatures observed in our shock experiments should be close to the melting temperature of the sample at the release pressure.

Figure 4 illustrates only one of several possible release paths involving melting. For example, if release melting were completed at a higher pressure than $P_{R}$ then the re- lease path would leave the melting curve and enter the melt field. Alternatively, at very high Hugoniot pressures where the shocked sample may be completely molten, the melting curve will not affect the release path. However, the sample will not be able to cool below the melting curve by thermal contact with the anvil until the heat of fusion is overcome, so the temperature is again buffered at about $T_{M}$. A complete discussion of various possible release and cooling paths is given by Tan and Ahrens [1990].

Just as the cold window can cause freezing of the sample, the hot sample can induce melting of the window material. In this case, a melt front will propagate into the window, a solidification front will propagate into the sample (Figure 5), and the energetics of both transitions will affect the observed interface temperature. This case has been treated in detail by Tan and Ahrens [1990] and is referred to in their paper as model III. Calculations of the melting curves and Hugoniot states for anvils and samples indicate that this model applies to the present experiments on stainless steel and most of the previous work on Fe samples.

The importance of the analysis of Tan and Ahrens [1990] is that it allows the observed interface temperature to be related to the melting temperature at the release pressure. For model III of these authors, the interface temperature is time independent and given by

$$
\begin{aligned}
& T_{I}=T_{W}+\frac{\alpha_{32} \operatorname{erf} \lambda}{\alpha_{32} \operatorname{erf} \lambda-\operatorname{erf} \mu}\left(T_{M}-T_{W}\right) \\
& T_{I}=T_{M}+\frac{\operatorname{erf} \mu}{\alpha_{32} \operatorname{erf} \lambda-\operatorname{erf} \mu}\left(T_{M}-T_{W}\right)
\end{aligned}
$$

where $T_{W}$ and $T_{M}$ are melting temperatures of the window and sample, respectively, and $\alpha_{32}$ is defined as in equation (4), but with respect to the solid metal and liquid anvil (see Figure 5). The rate parameters $\lambda$ and $\mu$ are related to the position $X$ of the melting front in the window and freezing front in the metal:

$$
\begin{aligned}
& X_{W}=2 \lambda\left(\kappa_{2} t\right)^{1 / 2} \\
& X_{M}=2 \mu\left(\kappa_{3} t\right)^{1 / 2}
\end{aligned}
$$

where subscripts 2 and 3 refer to molten window and solid metal (Figure 5). The method of solving for $\lambda$ and $\mu$ is given by Tan and Ahrens [1990]. An observed interface temperature $T_{I}$ is used to obtain $T_{M}$ by

$$
T_{M}=T_{W}+\left(T_{I}-T_{W}\right)\left(\frac{\operatorname{erf} \mu}{\alpha_{32} \operatorname{erf} \lambda}\right)
$$

\section{Stainless Steel Data}

The above analysis has been applied to the data obtained on our four stainless steel experiments. These four data represent all of our experimental results at this stage: we have not selectively culled the data or rejected any points. Thermal properties are calculated using the methods given by Tan and Ahrens [1990], and we also adopt their values for the material properties of the anvils. The Debye temperature of stainless steel under room conditions, which is needed to scale thermal properties, is taken from $M c Q u e e n$ et al. [1970], whereas the average of the liquidus and solidus temperatures (1699 K) [Lewis, 1977] is used for the melting temperature of 304 stainless. A constant value of $\rho \gamma=15$ is 


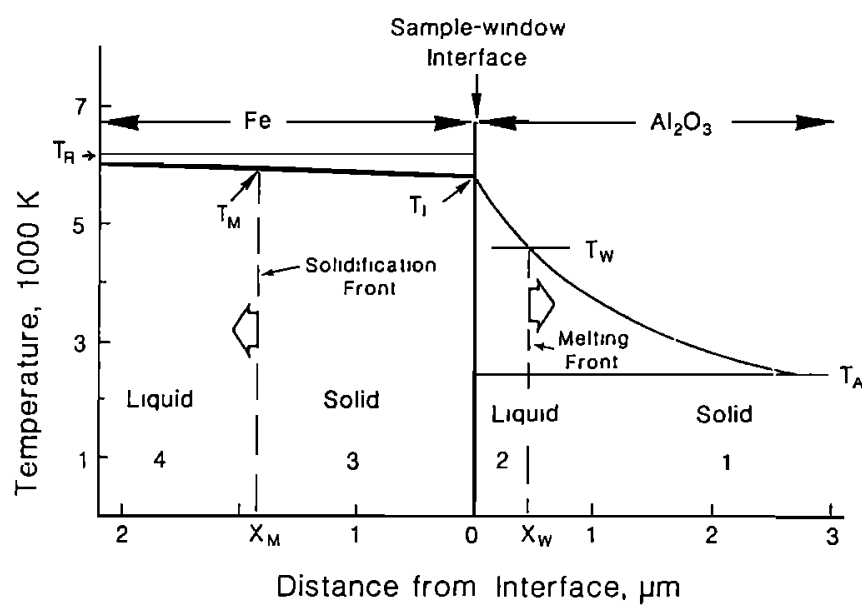

Fig. 5. Temperature as a function of position in the target for model III of Tan and Ahrens [1990]. The sample is originally a partial or complete melt which freezes due to contact with the anvil. The anvil is likewise melted due to contact with the hot sample. Note that the interface temperature $T_{I}$ is close to the melting point of the sample $T_{M} . T_{R}$ is the initial release temperature in the sample, and $T_{A}$ is the Hugoniot temperature of the anvil. The thermal profile is calculated for an Fe sample and sapphire anvil $0.1 \mu \mathrm{s}$ after arrival of the shock at the sample-anvil interface $(X=0)$ [after Tan and Ahrens, 1990].

assumed, similar to that for $\mathrm{Fe}$ [Brown and McQueen, 1986]. All other thermodynamic properties are taken as equal to those used in the earlier work on Fe [Tan and Ahrens, 1990]. We thus obtain the melting temperature $T_{M}$ of stainless steel at the release pressures defined by the shock impedence of the anvil materials. The inferred $T_{M}$ values are listed in Table 2 and are plotted in Figure 6. These values define a melting curve with a positive Clapyron slope. For the present, we ignore the difference between the liquidus and solidus, which at one atmosphere is $\sim 100 \mathrm{~K}$ and beyond the resolution of our experiments. It is significant that the datum obtained using a LiF window is entirely consistent with the trend for shots using $\mathrm{Al}_{2} \mathrm{O}_{3}$ windows. Because $\mathrm{LiF}$ and $\mathrm{Al}_{2} \mathrm{O}_{3}$ attain very different temperatures at high shock pressures [Svendsen et al., 1989], the internal consistency of these data strongly suggests that we are observing the temperature of the sample surface and that the windows are remaining transparent. If this were not true, the $\mathrm{LiF}$ window would yield a substantially higher temperature than the $\mathrm{Al}_{2} \mathrm{O}_{3}$ window.

It should be emphasized that the analysis of $\operatorname{Tan}$ and Ahrens [1990] relates the interface temperature only to melting at the release pressure, $T_{M}$. These authors concluded that it is possible to extract the Hugoniot temperature only at low shock pressures, where release melting does not occur, or at the highest pressures where a metal is completely melted and heat conduction to the anvil is not sufficient to induce solidification. In such cases, equations (2)-(4) are appropriate and $T_{H}$ can be extracted. However, it is apparent from Figure 4 that the two effects of release melting partly offset each other: Relative to simple release and heat conduction, in the absence of melting or freezing, the temperature drop due to release melting is greater (because melt is produced), whereas the temperature drop due to contact with the window is smaller (because of the latent heat of fusion). The compensating nature of these effects suggests that the simpler analysis of Grover and Urtiew [1974], which ignores melting effects, may actually be a reasonable approximation of $T_{H}$. To investigate this possibility, we have calculated theoretical interface temperatures by both methods, using $\mathrm{Fe}$ and stainless steel samples on $\mathrm{Al}_{2} \mathrm{O}_{3}$ and $\mathrm{LiF}$ windows as examples. The simulations show that for a given $P_{H}$, the interface temperatures which ignore melting, $T_{i}$, can be either greater or less than the actual interface temperature $T_{I}$, depending on the properties of the sample and the amount of release melting that occurs. If $T_{i}<T_{I}$, then using the Grover-Urtiew analysis for the higher observed interface temperature will overestimate $T_{H}$, while if $T_{i}>T_{I}$ the opposite holds true. The magnitude of the errors is approximately $200 \mathrm{~K}$ or less for conditions similar to those of previous experiments on Fe [Bass et al., 1987; Ahrens et al., $1990 b]$, and the present experiments. Applying these results to our stainless steel data, Hugoniot temperatures calculated from equations (2)-(4) for the three lowest-pressure shots $(214,218,219)$ should place lower bounds on the true Hugoniot temperatures; for the highest-pressure shot (215), the calculated Hugoniot temperature is an upper bound.

Thus far it has been implicitly assumed that an equilibrium state is achieved upon both shock release and conductive cooling of the sample. There is no absolute assurance that this condition is met, and it is possible that superheated solids or supercooled melts persist outside of their stability fields. In such a situation, equilibrium phase boundaries would have less effect on release and cooling paths than indicated by the calculations of Tan and Ahrens [1990], and the analysis of Grover and Urtiew [1974] could be more appropriate for obtaining Hugoniot temperatures.

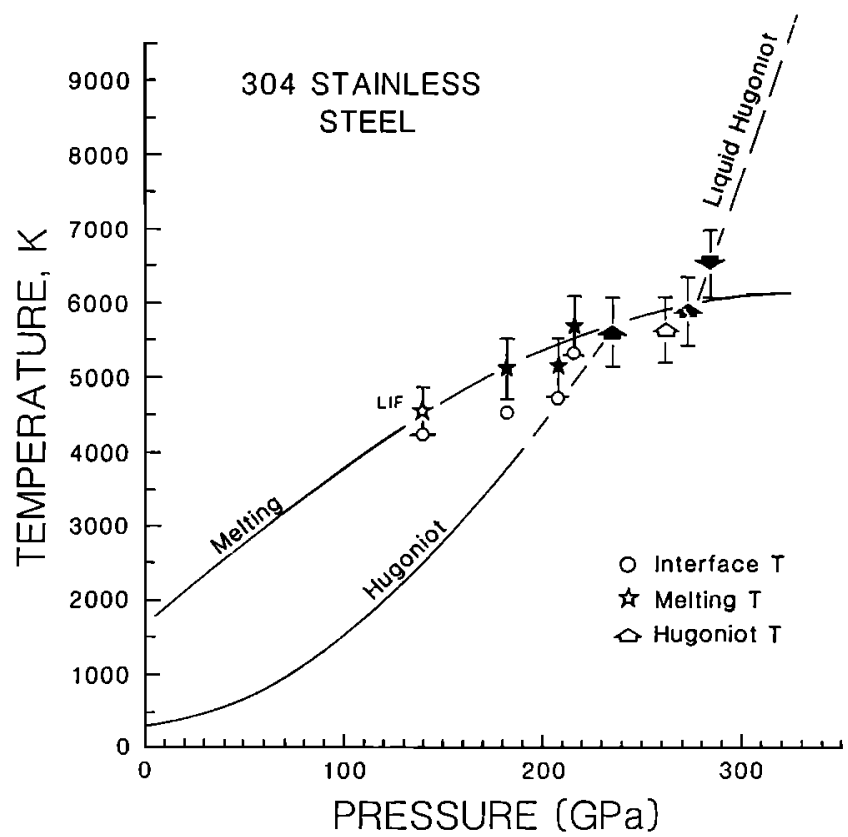

Fig. 6. Data from this study on 304 stainless steel. From the raw interface temperatures (circles), points on the melting curve of the metal (stars) are obtained using the analysis of Tan and Ahrens [1990]. Arrows are calculated using the analysis of Grover and Urtiew [1974], which ignores melting effects, and provide bounds on the Hugoniot temperatures $T_{H}$. The arrows point in the direction of $T_{H}$. Solid symbols represent shots using $\mathrm{Al}_{2} \mathrm{O}_{3}$ windows; open symbols indicate use of a $\mathrm{LiF}$ window. Theoretical values of $T_{H}$ [McQueen et al., 1970] are given by the solid curve; the dashed Hugoniot is inferred based upon the present study. 
Taken together, the data plotted in Figure 6 define an internally consistent trend for the melting curve of stainless steel. The fact that the inferred Hugoniot temperatures appear to extend the trend of the release melting temperatures indicates that the lower-pressure Hugoniot data are on the melting curve. Moreover, the datum at $234 \mathrm{GPa}$ and $5600 \mathrm{~K}$ is in excellent agreement with the calculated Hugoniot by McQueen et al. [1970], suggesting that the Hugoniot intersects the melting curve near this pressure. The highestpressure datum at $283 \mathrm{GPa}$ and $6580 \mathrm{~K}$ falls above the melting curve, and is likely a point on the liquid Hugoniot. The onset of melting along the Hugoniot at approximately 234 $\mathrm{GPa}$ and $5600 \mathrm{~K}$ is comparable with the values of $243 \mathrm{GPa}$ and $\sim 5000 \mathrm{~K}$ obtained for $\mathrm{Fe}$ on the basis of sound speed measurements [Brown and McQueen, 1986]. However, the pressure range over which the Hugoniot and melting curve coincide appears to be much greater for stainless steel than for Fe (using the data of Bass et al. [1987] and Williams et al. [1987]). Presumably, melting along the Hugoniot initiates at a solidus temperature $(234 \mathrm{GPa}$ and $5600 \mathrm{~K}$ in this case), whereas the Hugoniot enters the totally molten region at a liquidus temperature ( $\approx 271 \mathrm{GPa}$ and $5860 \mathrm{~K}$ ).

A comparison of the stainless steel data with previous experimental studies on the melting of Fe is shown in Figure 7. The hatchured area for Fe melting is bounded on the lower side by the curve of Williams et al. [1987], which is based on shock temperature measurements [Bass et al., 1987], and static measurements in a laser-heated diamond anvil cell. The upper bound is based on a reanalysis of the shock temperature data alone, taking into account release melting [Ahrens et al., 1990b]. The differences between these curves stem largely from the choice of data used in each analysis. Williams et al. [1987] obtained a curve consistent with the shock wave data that yielded the lowest Hugoniot temperatures [Bass et al., 1987], and which is also consistent with the static compression data of Williams et al. Five of the 13 shock wave data of Bass et al. [1987] were used. The rational for rejecting data was that any imperfections in the samples would likely result in an overestimation of the Hugoniot temperature and that the lowest temperature data should best approximate the true Hugoniot temperatures. In contrast, Ahrens et al. [1990] used an energy balance consideration to decide if a portion of the thermal radiation in the shock temperature experiments was spurrious. These authors used eight of the data from Bass et al. [1987]. If the analysis of Ahrens et al. is limited to the five lower temperature data used by Williams et al. [1987] and Bass et al. [1987], then the resultant melting curve is at lower temperatures and is in significanty better agreement with the Williams et al. curve. In the following discussion we use the melting curve of Williams et al. [1987] as a basis for comparison with our new data because we feel that our original criterion for assessing the shock data is more stringent. The melting curve of Williams et al. represents a higher degree of internal consistency between the shock wave and static compression data. In addition, their Hugoniot temperatures for liquid $\mathrm{Fe}$ are in better agreement with those calculated by Anderson [1990].

The melting curves of Williams et al. [1987] and Ahrens et al. [1987] are in poor agreement with that determined for Fe to $\sim 114 \mathrm{GPa}$ by Boehler [1986] and Boehler et al. [this issue]. The latter melting curve is based upon optical pyrometric measurements on resistively heated wires and

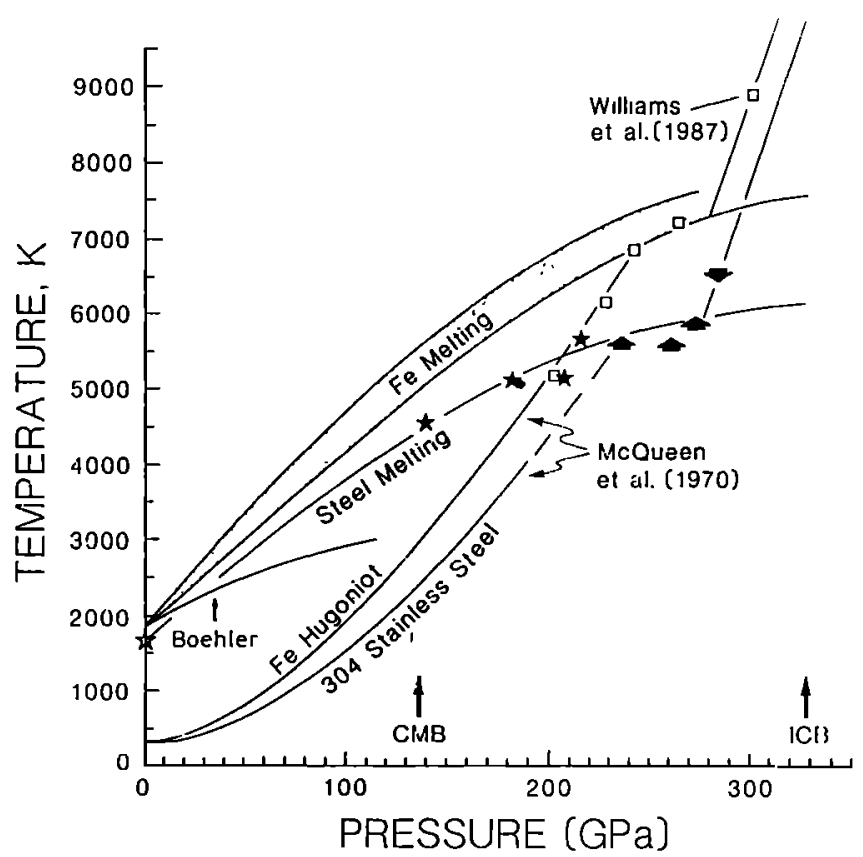

Fig. 7. A comparison of the results from this study with previous measurements on Fe. Hatchured area for Fe melting is defined by the results of Bass et al. [1987], Williams et al. [1987], and Ahrens et al. [1990b]. The melting curve labelled "Boehler" is from the work of Boehler [1986] and Boehler et al. [this issue].

laser heated foils in a diamond cell. A detailed comparison of the available static compression work is beyond the scope of this paper (see Williams et al. [1990], and Boehler et al. [this issue]), but it is fair to state that the reasons for the discrepancies among the data are currently unresolved. For the present we simply point out the internal consistency between the shock and static compression data of Williams et al. [1987] and that the melting data of Boehler [1986] and Boehler et al. [this issue] seem to require the existance of a new, as yet unidentified phase in order to reconcile their phase diagram for $\mathrm{Fe}$ with Hugoniot sound velocity measurements [Brown and McQueen, 1986].

Compared with the Fe Hugoniot temperatures of Bass et al. [1987], the stainless steel data indicate lower shock temperatures at all pressures. This behavior is expected on the basis of the higher bulk modulus of stainless steel, and is supported by theoretical calculations of shock temperatures [McQueen et al., 1970]. Moreover, the Fe melting curve of Williams et al. [1987] is subparallel to the melting curve of stainless steel, which is not surprising given the high Fe content of the steel and similarity in the properties of $\mathrm{Fe}, \mathrm{Cr}$, and $\mathrm{Ni}$. If we consider melting temperatures, the results of Williams et al. [1987], indicate that the melting point of $\mathrm{Fe}$ at the pressure of the inner core-outer core boundary (ICB) is $7600 \pm 500 \mathrm{~K}$. By comparison, stainless steel with $\approx 20 \%$ $\mathrm{Cr}$ and $\approx 10 \% \mathrm{Ni}$ melts at a temperature lower by approximately $1450 \mathrm{~K}$ (Figure 7), which is substantially greater than the difference of $\sim 100 \mathrm{~K}$ between the 1-atm melting points. Thus these studies indicate that the addition of $\mathrm{Ni}$ and/or $\mathrm{Cr}$ to $\mathrm{Fe}$ yields a melting point depression of Fe which increases dramatically with increasing pressure. In contrast, a comparison of the stainless steel data from the present work with the Fe melting curve of Boehler et al. [this issue] would indicate that the melting curves of these mate- 
rials cross at pressures of $\sim 20-40 \mathrm{GPa}$, and that at higher pressures the stainless steel melting temperature is greater than that of Fe. Although neither of these possibilities can be ruled out with certainty, it would be an interesting surprise if the melting point of Fe were elevated at high $P$ by the addition of $\mathrm{Ni}$ and/or $\mathrm{Cr}$. Not only would this behavior be in marked contrast to the one atmosphere melting point depression, but the data of Urakawa et al. [1987] on the Fe-Ni-S-O system up to $15 \mathrm{GPa}$ shows evidence for a melting point depression, relative to the $\mathrm{Ni}$-free system, at all pressures investigated.

Cosmochemical and meteoritic evidence suggests that the core contains approximately 5 wt \% Ni [Ringwood, 1977; Brett, 1976], whereas $\mathrm{Cr}$ is thought to be far less abundant. Although our results, as well as those of previous studies (e.g., Urakawa et al. [1987]), imply that the melting point of $\mathrm{Fe}$ is significantly depressed at core pressures by alloying with $\mathrm{Ni}$ and $\mathrm{Cr}$, it is unfortunately not possible with our data on stainless steel to decouple the effects of $\mathrm{Ni}$ and $\mathrm{Cr}$. Clearly, further work is required to quantify the melting relations for $\mathrm{Fe}$ alloys. Therefore, the value of $7600 \pm 500$ $\mathrm{K}$ for the melting point of $\mathrm{Fe}$ at the inner core-outer core boundary [Williams et al., 1987] remains a reasonable upper bound on the temperature at that depth.

Acknowledgments. We thank Q. Williams R. Jeanloz, R.G. McQueen, D. Isaak, R. Boehler, and A. Chopelas for preprints of their work. E. Gelle, M. Long, and K. Gallagher provided critical assistance in conducting these experiments. We appreciate the comments of two anonymous reviewers on an earlier version of this work. We thank the Max Planck Institut für Chemie for assistance with the preparation of this manuscript. This research was supported by NSF grants to the University of Illinois and the California Institute of Technology. Contribution 4895, California Institute of Technology.

\section{REFERENCES}

Ahrens, T.J., Shock wave techniques for geophysics and planetary physics, Methods Exp. Phys., 24, 185-235, 1987.

Ahrens, T.J., J.D. Bass and J.R. Abelson, Shock temperatures in metals, in Shock Compression of Condensed Matter, 1989 , edited by S.C. Schmidt, J.N. Johnson and L.W. Davison, pp. 851-858, North-Holland, Amsterdam, 1990a.

Ahrens, T.J., H. Tan and J.D. Bass, Analysis of shock temperature data for iron, High Pressure Res., 2, 145-157, 1990b.

Anderson, W.W., High Pressure States in Condensed Matter: I. High Pressure Behavior of the Iron-Sulfur System With Applications to the Earth's Core, Ph.D. dissertation, Cal. Inst. Tech., Pasadena, 1990.

Ashcroft, N.W. and N.D. Mermin, Solid State Physics, 826 pp., Holt, Rinehart and Winston, Philadelphia, Pa., 1976.

Bass J.D., B. Svendsen, and T.J. Ahrens, The temperature of shock compressed iron, in High Pressure Research in Mineral Physics, Geophys. Monogr. Ser., vol. 39, edited by M.H. Manghnani and Y. Syono, pp. 393-402, AGU, Washington, D.C., 1987.

Boehler, R., The phase diagram of iron to $430 \mathrm{kbar}$, Geophys. Res. Lett., 19, 1153-1156, 1986.

Boehler, R., N. von Bargen, and A. Chopelas, Melting, thermal expansion and phase transitions of iron at high pressures, $J$. Geophys. Res, this issue.

Boness, D.A., J.M. Brown, and A.K. McMahan, The electronic thermodynamics of iron under Earth core conditions, Phys. Earth Planet. Inter., 42, 227-240, 1986.

Boslough, M.B., and T.J. Ahrens, A sensitive time-resolved radiation pyrometer for shock-temperature measurements above 1500K, Rev. Sci. Instrum., 60, 3711-3716, 1989.

Brett, R., The current status of speculations on the composition of the core of the Earth, Rev. Geophys., 14, 375-383, 1976.

Brown, J.M., and R.G. McQueen, Phase transitions, Grüneisen parameter, and elasticity for shocked iron between $77 \mathrm{GPa}$ and $400 \mathrm{GPa}, J$. Geophys. Res., 91, 7485-7494, 1986.

Carter, W.J., Hugoniot equation of state of some alkali halides, High Temp. High Pressures, 5, 313-318, 1973.

Dziewonski, A.M., and D.L. Anderson, Preliminary reference Earth model, Phys. Earth Planet. Inter., 25, 297-356, 1981.

Grover, R., and P.A. Urtiew, Thermal relaxation at interfaces following shock compression, J. Appl. Phys., 45, 146-152, 1974.

Hansen, M., and K. Anderko, Constitution of Binary Alloys, 1305 pp., McGraw-Hill, New York, 1958.

Jeanloz, R., and T.J. Ahrens, Pyroxenes and olivines: structural implications of shock-wave data for high-pressure phases, in High-Pressure Research: Applications to Geophysics, edited by M.H. Manghnani ahd S. Akimoto, pp. 439-461, Academic, San Diego, Calif., 1977.

Keeler, R.N., Electrical conductivity of condensed media at high pressures, in Physics of High Energy Density, Proc. Int. Sch. Phys. Enrico Fermi XLVIII, edited by P. Caldirola and $\mathrm{H}$. Knoepfel, pp. 106-125, Academic, San Diego, Calif., 1971.

Lewis, J.R., Physical properties of stainless steels, in Handbook of Stainless Steels, edited by D. Peckner and I.M. Bernstein, pp. 19-1-19-36, McGraw-Hill, New York, 1977.

Lyzenga, G.A., and T.J. Ahrens, The relation between the shockinduced free-surface velocity and the postshock specific volume of solids, J. Appl. Phys., 49 201-204, 1978.

Lyzenga, G.A., and T.J. Ahrens, Multiwavelength optical pyrometer for shock compression experiments, Rev. Sci. Instrum., 50 , 1421-1424, 1979.

Marsh, S.P. (Ed.), LASL Shock Hugoniot Data, pp. 260-263, University of California Press, Berkeley, 1980.

Matassov, G., The Electrical Conductivity of Iron-Silicon Alloys at High Pressures and the Earth's Core, Ph.D. thesis, Univ. of Calif., Livermore, 1977.

McQueen, R.G., and D.G. Isaak, Characterizing windows for shock-wave radiation studies, J. Geophys. Res., this issue.

McQueen, R.G., S.P. Marsh, J.W. Taylor, J.N. Fritz, and W.J. Carter, The equation of state of solids from shock wave studies, in High Velocity Impact Phenomena, edited by R. Kinslow, Pp. 293-417, Academic, San Diego, Calif, 1970.

Mitchell, A.C., and W.J. Nellis, Shock compression of aluminum, copper and tantalum, J. Appl. Phys., 52, 3363-3375, 1981.

Rice, M.H., R.G. McQueen, and J.M. Walsh, Compression of solids by strong shock waves, Solid State Physics, 6, 1-63, 1958.

Ringwood, A. E., Composition of the core and implications for origin of the Earth, Geoch em. J., 11, 111-135, 1977.

Roufosse, M.C., and R. Jeanloz, Thermal conductivity of minerals at high pressure: The effect of phase transitions, J. Geophys. Res., 88, 7399-7409, 1983.

Schmitt, D.R., and T.J. Ahrens, Emission spectra of shock compressed solids, in Shock Waves In Condensed Matter, 1989, edited by J.R. Asay, R.A. Graham, and G.K. Straub, Pp. 313316, Elsevier, New York, 1984.

Svendsen, B., J.D. Bass, and T.J. Ahrens, Optical radiation from shock-compressed materials and interfaces, Phys. Rep., 180, 333-416, 1989.

Tan, H., and T.J. Ahrens, Shock temperature measurements for metals, High Pressure Res., 2, 159-182, 1990.

Urakawa, S., M. Kato, and M. Kumazawa, Experimental study on the phase relations in the system $\mathrm{Fe}-\mathrm{Ni}-\mathrm{O}-\mathrm{S}$ up to $15 \mathrm{GPa}$, in High Pressure Research in Mineral Physics, Geophys. Monogr. Ser., vol. 39, edited by M.H. Manghnani and Y. Syono, PP. 95111, AGU, Washington, D.C., 1987.

Urtiew, P.A., Effect of shock loading on transparency of sapphire crystals, J. A ppl. Phys., 45, 3490-3493, 1974.

Urtiew, P.A., and R. Grover, Temperature deposition caused by shock interactions with material interfaces, J. Appl. Phys., 45, 140-145, 1974.

Urtiew, P.A., and R. Grover, The melting temperature of magnesium under shock loading, J. Appl. Phys., 48, 1122-1126, 1977.

Williams, Q., R. Jeanloz, J. Bass, B. Svendsen, and T.J. Ahrens, The melting curve of iron to 250 gigapascals: A constraint on the temperature at Earth's center, Science, 236, 181-182, 1987.

Williams, Q. , E. Knittle, and R. Jeanloz, The high-pressure melting curve of iron: A technical discussion, J. Geophys. Res., in press, 1990. 
J.R. Abelson, Coordinated Sciences Laboratory, University of Illinois, Urbana, IL 61801

T.J. Ahrens, Seismological Laboratory, California Institute of Technology, Pasadena, CA 91125.

J.D. Bass, Department of Geolozy, University of Ilinois, 1301 W. Green St., Urbana, IL 61801
T. Hua, Beijing Institute of Technology, P. O. 327, Beijing, Peoples Republic of China.

(Received July 23, 1990;

revised September 28, 1990; accepted October 14, 1990.) 